\title{
Tradeoffs Between Effectiveness and Social Perception When Using Mixed Reality to Supplement Gesturally Limited Robots
}

\author{
Jared Hamilton \\ jghamilton@mines.edu \\ MIRRORLab \\ Colorado School of Mines \\ Golden, Colorado
}

\author{
Nhan Tran \\ nttran@mines.edu \\ MIRRORLab \\ Colorado School of Mines \\ Golden, Colorado
}

\author{
Tom Williams \\ twilliams@mines.edu \\ MIRRORLab \\ Colorado School of Mines \\ Golden, Colorado
}

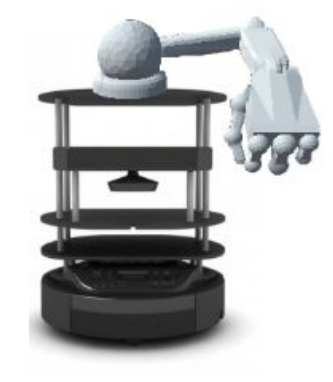

\begin{abstract}
Mixed reality visualizations provide a powerful new approach for enabling gestural capabilities for non-humanoid robots. This paper explores two different categories of mixed-reality deictic gestures for armless robots: a virtual arrow positioned over a target referent (a non-ego-sensitive allocentric gesture) and a virtual arrow positioned over the robot (an ego-sensitive allocentric gesture). We explore the trade-offs between these two types of gestures, with respect to both objective performance and subjective social perceptions. We conducted a 24-participant within-subjects experiment in which a HoloLens-wearing participant interacted with a robot that used these two types of gestures to refer to objects at two different distances. Our results demonstrate a clear trade-off between performance and social perception: non-ego-sensitive allocentric gestures led to quicker reaction time and higher accuracy, but ego-sensitive gesture led to higher perceived social presence, anthropomorphism, and likability. These results present a challenging design decision to creators of mixed reality robotic systems.
\end{abstract}

\section{KEYWORDS}

Augmented Reality, Mixed Reality, Deictic Gesture, Social Presence, Anthropomorphism, Human-Robot Interaction

\section{INTRODUCTION}

For robots to be able to communicate effectively with humans they must be able to engage in natural, human-like human-robot dialogue $[7,21,30]$. In contrast to the dialogue agents and chatbots developed in the field of natural language processing, interactive robots must be able to communicate with increased sensitivity to their situated context [21,34]. We have argued across our work that this requires three broad competencies: environmental context sensitivity (sensitivity to the spatially situated, large-scale, uncertain, and incompletely known nature of realistic task environments [39]); cognitive context sensitivity (sensitivity to the working memory

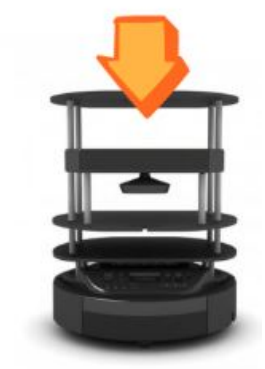

and attentional constraints of realistic teammates [40]); and social context sensitivity (sensitivity to the relational context into which they are embedded, and the importance of strengthening and maintaining social relationships through adherence to social and moral norms $[18,42]$ and active maintenance and repair of trust and rapport $[13,16,25])$.

Critically, for these three competencies to be mastered, robots must be able not only to understand and generate appropriate verbal behavior, but also to understand and generate appropriate accompanying nonverbal behaviors such as gesture and eye gaze. Not only are nonverbal behaviors critical for situated interaction $[2,12,14,23]$, but it is integrally related to each of these three competencies. Deictic gestures such as pointing inherently leverage environmental context by identifying nearby referents (typically, cp. [31]), especially when such referents are not currently known or attended to by interlocutors; these gestures are often made due to cognitive context, in order to direct interlocutor attention [19] and reduce memory costs that would be imposed by communication $[10,23]$; and gestures are often generated in ways that mimic those of interlocutors, in order to increase engagement and build rapport through mirroring [6]. As such it is no surprise that roboticists have been seeking to enable nonverbal competence to reap these same benefits $[1,4,5,24,27-29]$

Unfortunately generation of human-like gestures and eye gaze are not available to all robots due to differences in morphology; many if not most robotic platforms lack the arms, heads, and eyes needed to generate expressive cues. This is especially true for mobile bases such as those used in warehouses, and free-flying drone platforms. While these types of robots may not be designed to be sociable, they still need gaze- and gestural-capabilities to communicate about objects with teammates. Accordingly, researchers have been investigating new methods for nonverbal signalling (e.g., directed lighting cues) that may achieve the same communicative goals typically addressed by physical gaze and gesture [9,32]. 
Mixed-reality technologies such as the Microsoft HoloLens stand to enable exciting new approaches for generating gaze and gestural cues in this vein for robots with non-humanoid morphologies. The space of visualizations used as mixed-reality deictic gestures (which can altogether be classified as view-augmenting mixed reality interaction design elements in the Reality-Virtuality Interaction Cube framework of Williams, Szafir, and Chakraborti [41]) can be divided into at least five primary classes: allocentric gestures (e.g., circling a target referent in a user's augmented reality head-mounted display (AR-HMD)), perspective-free gestures (e.g., projecting a circle around a target referent on the floor of the shared environment), ego-sensitive allocentric gestures (e.g., pointing to a target referent using a simulated arm rendered in a user's AR HMD), and egosensitive perspective-free gestures (e.g., projecting a line from the robot to its target on the floor of the shared environment Williams et al. [43]. In previous work, Williams et al. specifically investigated the first of these categories, allocentric gestures, and demonstrated that such mixed-reality gestures can significantly increase the communicative effectiveness of non-humanoid robots [35, 37, 38].

One downside of these previous explorations of allocentric gesture is the low ecological validity of the context in which they were assessed, with crowdworkers viewing interactive videos simulating the expected appearance of such gestures. One consequence of this is that participants in those previous experiments had full fieldof-view and viewed the entire experimental environment through an unchanging vantage point. In realistic task contexts, users are unlikely to be able to view their entire task environment from a single perspective, and mixed reality deictic gestures must be delivered through platforms like the HoloLens, which severely restrict the portion of the environment in which such gestures can be displayed. We predict that in even moderately larger task contexts, these factors will result in users completely directing their field of view towards the regions in which mixed-reality deictic gestures are being displayed, and will be able to completely avoid directing their visual attention back towards the non-humanoid robot who is generating those visualizations in the first place. We further predict that this lack of attention towards the robot could have detrimental long-term effects on human-robot teaming, such as decreased trust, rapport, and situation awareness.

These challenges may be addressable by another form of mixed reality deictic gesture highlighted in Williams et al. [43]'s taxonomy: ego-sensitive allocentric gestures, in which simulated arms are rendered above the non-humanoid robot, and used to point at target referents just as physical arms would [see, e.g., 15]. We would expect the use of such arms to increase the robot's anthropomorphism, and because users would need to consistently look towards the robot to see where it is pointing, it would likely also enjoy increased social presence, potentially preventing against the aforementioned predicted long-term consequences of non-ego-sensitive allocentric gesture.

On the other hand, ego-sensitive allocentric gestures may come with their own challenges. Specifically, because users will need to follow the vector along which the robot is pointing, and estimate for themselves which objects fall within the robot's deictic cone, they may be less accurate and efficient at determining the targets of these gestures, especially when target referents are far from the robot (the very context in which ego-sensitive allocentric gestures are expected to provide social benefits).

In this paper, we present an experiment to systematically evaluate these expected differences in social- and task-oriented benefits between ego-sensitive and non-ego-sensitive forms of allocentric gesture, as well as the impact of target distance on these differences. The remainder of the paper proceeds as follows: in Section 2 we formally define our experimental hypotheses; in Section 3 we describe the design of a human-subject experiment designed to analyze those hypotheses; in Section 4 we present the results of that experiment; and in Section 5 we discuss our results and suggest directions for future work.

\section{HYPOTHESES}

Our experiment assesses two key hypotheses:

H1: We hypothesized that a robot that uses non-ego-sensitive allocentric gestures (i.e., arrows drawn over target referents) when referring to target referents will:

(H1.1) be more effective than a robot using ego-sensitive allocentric gestures (i.e., pointing using virtual arms) as measured by (1) accuracy and (2) reaction time, and

(H1.2) that these benefits would be more pronounced for objects farther away from the robot.

H2: We hypothesized that a robot that uses non-ego-sensitive allocentric gestures (i.e., arrows drawn over target referents) when referring to target referents will:

(H2.1) have lower social perception than a robot using egosensitive allocentric gestures (i.e., pointing using virtual arms) as measured by (1) social presence, (2) anthropomorphism, (3) likability, (4) warmth, and (5) perceived competence

(H2.2) that these detriments would be more pronounced for objects farther away from the robot.

\section{EXPERIMENT}

To test these hypotheses, we designed a within-subjects humansubject study in which HoloLens-equipped participants interacted with a mobile robot that used two types of mixed-reality deictic gestures. All aspects of our experimental design received IRB approval.

\subsection{Task Design}

In each trial, participant interacted with a Kabuki Turtlebot (Fig. 1) who was positioned three meters away from the participant. Affixed to the top of the Turtlebot was an AR Cube: a small cardboard cube with fiducial markers on each face of the cube.

Each participant was given a Microsoft HoloLens to wear. When viewing the scene through the HoloLens, participant was able to perceive a row of three spheres (red, green, and blue) hovering a half-meter above the ground, between the subject and the Turtlebot. During each experimental block, the Turtlebot gestures to one of these balls, and the participant was required to air-click on it using a HoloLens-recognized gesture. This pattern was repeated ten times, with the Turtlebot gesturing towards a randomly selected ball in each of the ten trials within the block. 


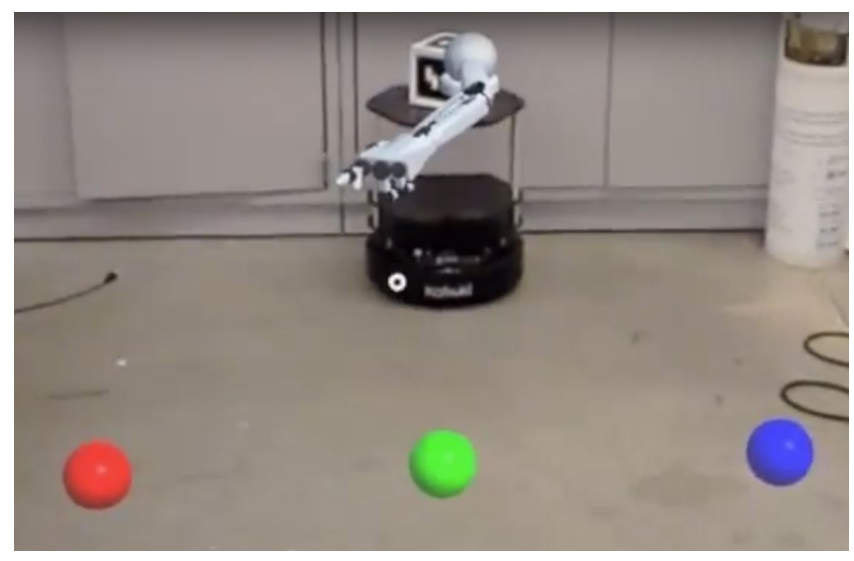

Figure 1: Robot arm gesturing to holographic sphere (Not in experimental environment).

\subsection{Experimental Design}

Each participant participated in four order-counterbalanced blocks of interactions with the mobile robot, with each block corresponding with a different setting of two two-level independent variables.

Our first independent variable was gesture type. In two of the four within-subject blocks, (the arm conditions), the robot with which participants interacted gestured toward the spheres using an egosensitive allocentric gesture: a virtual arm was visible on top of the robot, as shown in Fig. 1, which reached out and pointed towards each target sphere within the block. In the other two within-subject blocks, (the arrow conditions), the robot with which participants interacted gestured toward the spheres using a non-ego-sensitive allocentric gesture: an arrow appeared over each target sphere within the block.

Our second independent variable was target distance. In two of the four within-subject blocks, (the close conditions), the spheres were positioned approximately one meter from the robot and two meters from the human. In the other two within-subject blocks, (the far conditions), the spheres were positioned approximately two meters from the robot and one meter from the human.

Each participant participated in four ten-trial blocks, each associated with a different combination of these two two-level independent variables, with block ordering counterbalanced across participants.

\subsection{Measures}

This experimental design was used to assess the impact of our two independent variables on seven dependent variables, assessed using the following measures.

3.3.1 Objective Measures. Our first hypothesis was assessed using two objective measures:

Accuracy was measured as the proportion of objects in each trial that the user correctly selected.

Reaction Time was measured as the time (in seconds) it took for the user to select an object after the Turtlebot's gesture had completed.
3.3.2 Subjective Measures. Our second hypothesis was assessed using five sets of survey questions administered after each experiment block. Each set of survey questions was a Likert scale comprised of 5-6 Likert items, each of which asked for agreement or disagreement with a statement on a 1-5 scale.

Social Presence was measured using the Almere Social Presence scale [17].

Anthropomorphism was measured using the Godspeed II Anthropomorphism scale [3].

Likeability was measured using the Godspeed II Likeability scale [3]. Warmth was measured using the RoSAS Warmth scale [8].

Competence was measured using the RoSAS Competence scale [8].

\subsection{Procedure}

Participants were recruited on campus through web postings and flyers. Upon arriving and providing informed consent and demographic information, participants were introduced to the TurtleBot and the HoloLens. Participants then ran through all four experiment blocks through a single HoloLens application. At the end of each experiment block, this application instructed participants to remove the headset and adjourn to a nearby survey table to complete the subjective questionnaires; at the end of each survey, participants returned to the HoloLens. This cycle repeated until the experiment completed.

\subsection{Participants}

24 participants were recruited ( $14 \mathrm{M}, 10 \mathrm{~F})$, ranging in age from 18 to $52(\mathrm{M}=22.46, \mathrm{SD}=7.86)$. 20 of the 24 had not previously engaged in any experiments from our laboratory involving mixed reality.

\subsection{Analysis}

Data analysis was performed within a Bayesian analysis framework using the JASP 0.8.5.1 [33] software package, using the default settings as justified by Wagenmakers et al. [36]. For each measure, a Bayesian repeated measures analysis of variance $[11,22,26]$ was performed, using gesture type and target distance as random factors. Baws factors [20] were then computed for each candidate main effect and interaction, indicating (in the form of a Bayes Factor) for that effect the evidence weight of all candidate models including that effect compared to the evidence weight of all candidate models not including that effect, i.e.

$$
\frac{\sum_{m \in M \mid e \in m} P(m \mid \text { data })}{\sum_{m \in M \mid e \notin m} P(m \mid \text { data })},
$$

where $e$ is an effect under consideration, and $m$ is a candidate model in the space of candidate models $M$.

\section{RESULTS}

\subsection{Hypothesis One}

We hypothesized that a robot that uses non-ego-sensitive allocentric gestures (i.e., arrows drawn over target referents) when referring to target referents will: (H1.1) be more effective than a robot using ego-sensitive allocentric gestures (i.e., pointing using virtual arms) as measured by (1) accuracy and (2) reaction time, and (H1.2) that these benefits would be more pronounced for objects farther away 


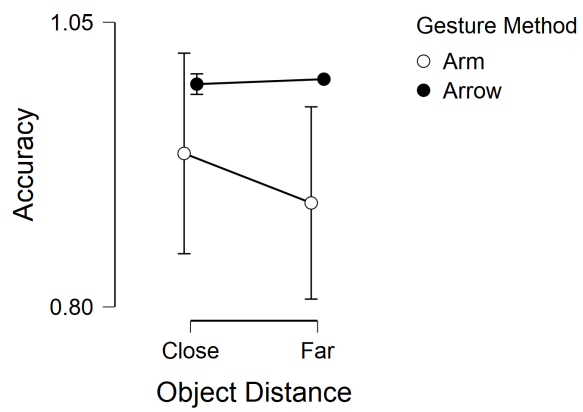

(a) Accuracy

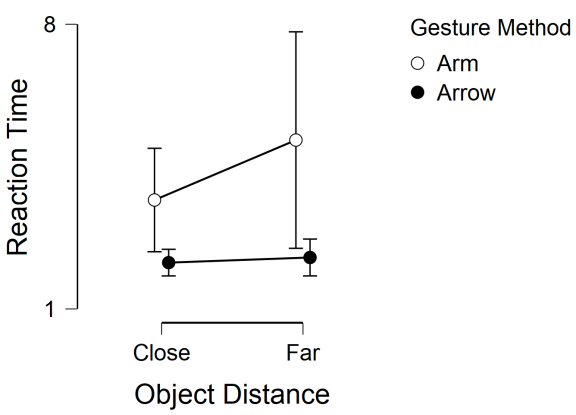

(b) Reaction Time

Figure 2: Objective Results

from the robot. We will thus separately assess this hypothesis for accuracy and for reaction time.

4.1.1 Accuracy. Our results provided strong evidence in favor of an effect of gesture type on accuracy (Bf 16.376) ${ }^{1}$, as shown in Fig. 2a, suggesting specifically that when non-ego-sensitive allocentric gestures were used, participants had higher accuracy rates. However, anecodtal evidence was found against an interaction effect between gesture type and referent distance on accuracy (Bf 2.41).

4.1.2 Reaction time. Our results provided strong evidence in favor of an effect of gesture type on reaction time (Bf 22.264), as shown in Fig. 2b, suggesting specifically that when non-ego-sensitive allocentric gestures were used, participants had faster reaction times. However, anecodtal evidence was found against an interaction effect between gesture type and referent distance on reaction time (Bf 1.98).

Overall these results support Hypothesis H1.1 but fail to support Hypothesis H1.2.

\subsection{Hypothesis Two}

We hypothesized that a robot that uses non-ego-sensitive allocentric gestures (i.e., arrows drawn over target referents) when referring to target referents will: (H2.1) be have lower social perception than a robot using ego-sensitive allocentric gestures (i.e., pointing using virtual arms) as measured by (1) social presence, (2) anthropomorphism, (3) likability, (4) warmth, and (5) perceived competence, and (H2.2) that these detriments would be more pronounced for objects farther away from the robot. We will thus separately assess this hypothesis for each of these subjective measures.

4.2.1 Social Presence. Our results provided extreme evidence in favor of an effect of gesture type on social presence (Bf 440.332), as shown in Fig. 3a, suggesting specifically that when non-egosensitive allocentric gestures were used, participants viewed the robot as having lower social presence. However, our results provided no significant evidence for or against of an interaction between gesture type and target distance on social presence, suggesting that more data must be collected before a conclusion can be reached.

\footnotetext{
${ }^{1}$ Our Bayes Factor of 16.376 suggests that our data were 16 times more likely to be generated under models in which gesture type is included than under those in which it is not.
}

Visual inspection of Fig. 3a suggests that it is entirely plausible that it is in fact when objects were close to the the robot that the arm achieved greater social presence; a surprising finding that would warrant further consideration if additional evidence were to reveal a statistically significant effect.

4.2.2 Anthropomorphism. Our results provided strong evidence in favor of an effect of gesture type on anthropomorphism (Bf 6026.6), as shown in Fig. 3b, suggesting specifically that when nonego-sensitive allocentric gestures were used, participants viewed the robot as having lower anthropomorphism. However, moderate evidence was found against an interaction effect between gesture type and referent distance on perceived anthropomorphism (Bf 3.32).

4.2.3 Likability. Our results provided moderate evidence in favor of an effect of gesture type on likability (Bf 6.145), as shown in Fig. 3c, suggesting specifically that when non-ego-sensitive allocentric gestures were used, participants viewed the robot as having lower likability. However, moderate evidence was found against an interaction effect between gesture type and referent distance on perceived likability (Bf 3.13).

4.2.4 Warmth. Our results provided no significant evidence for or against of an effect of gesture type on warmth (Bf 1.567), as shown in Fig. 3d, suggesting that more data must be collected before a conclusion can be reached. Moreover, moderate evidence was found against an interaction effect between gesture type and referent distance on perceived warmth (Bf 3.05).

4.2.5 Competence. Our results provided no significant evidence for or against of an effect of gesture type on competence (Bf 1.194), as shown in Fig. 3e, suggesting that more data must be collected before a conclusion can be reached. Moreover, moderate evidence was found against an interaction effect between gesture type and referent distance on perceived competence (Bf 3.52).

Overall these results support Hypothesis H2.1 but fail to support Hypothesis H2.2.

\section{CONCLUSION}

This paper sought to explore the objective and subjective differences between ego-sensitive and non-ego-sensitive allocentric mixed reality deictic gestures. As hypothesized, we discovered a dichotomy 


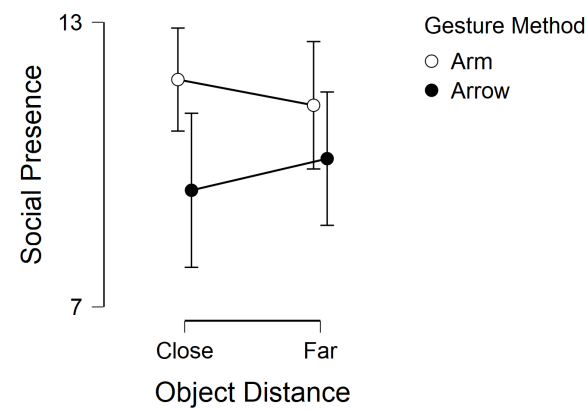

(a) Social Presence

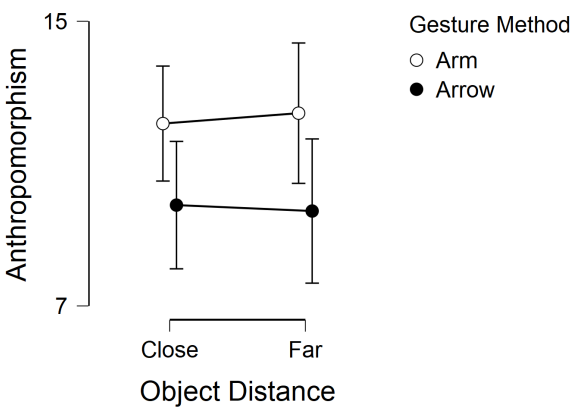

(b) Anthropomorphism

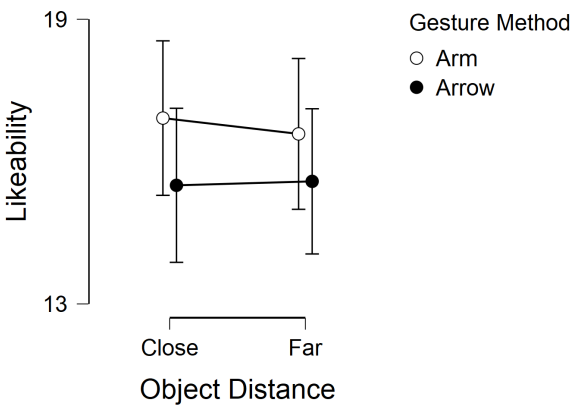

(c) Likability

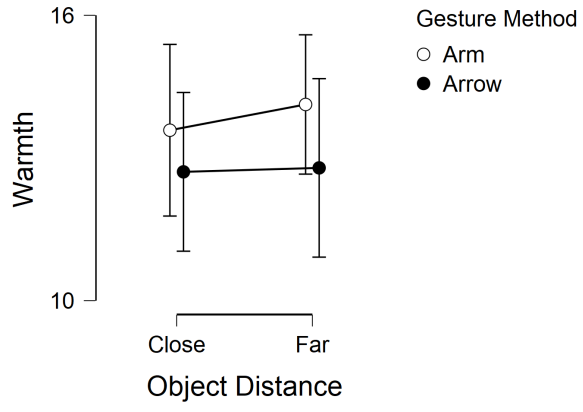

(d) Warmth

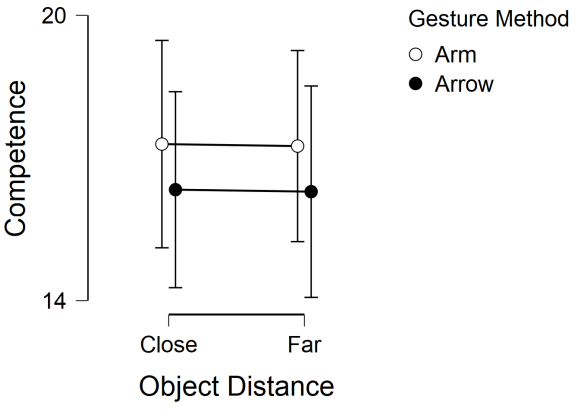

(e) Competence

Figure 3: Subjective Results

between these two gestural categories that presents a challenge for robot designers. Specifically, while ego-sensitive allocentric gestures such as pointing with virtual arms result in social benefits such as increased social presence, perceived anthropomorphism, and likability, non-ego-sensitive allocentric gestures such as virtual arrows result in greater task performance with respect to both speed and accuracy. In future work we plan to explore whether robots may achieve the "best of both worlds" by using both visualizations together, or whether this would be too cognitively overloading or perceived as too busy.

While our secondary distance-oriented hypotheses were not supported, for several of our objective and subjective measures our analyses were unable to directly support or refute these hypotheses, suggesting that more data must be collected before a decision can be made one way or another. Because our experiment was conducted using a Bayesian analysis framework, we are able to do just this, without violating a sampling plan or test assumptions, and thus plan to do so in future work.

\section{ACKNOWLEDGMENTS}

This work was funded in part by National Science Foundation grants IIS-1909864 and CNS-1810631.

\section{REFERENCES}

[1] Henny Admoni and Brian Scassellati. 2017. Social eye gaze in human-robot interaction: a review. Journal of Human-Robot Interaction 6, 1 (2017), 25-63.

[2] Adrian Bangerter and Max M Louwerse. 2005. Focusing attention with deictic gestures and linguistic expressions. In Proceedings of the Annual Meeting of the Cognitive Science Society, Vol. 27.
[3] Christoph Bartneck, Dana Kulić, Elizabeth Croft, and Susana Zoghbi. 2009. Measurement instruments for the anthropomorphism, animacy, likeability, perceived intelligence, and perceived safety of robots. International journal of social robotics 1, 1 (2009), 71-81.

[4] Cynthia Breazeal, Cory D Kidd, Andrea Lockerd Thomaz, Guy Hoffman, and Matt Berlin. 2005. Effects of nonverbal communication on efficiency and robustness in human-robot teamwork. In 2005 IEEE/RSf international conference on intelligent robots and systems. IEEE, 708-713.

[5] Andrew G Brooks and Cynthia Breazeal. 2006. Working with robots and objects: Revisiting deictic reference for achieving spatial common ground. In Proceedings of the 1st ACM SIGCHI/SIGART conference on Human-robot interaction. 297-304.

[6] Judee K Burgoon and Gregory D Hoobler. 1994. Nonverbal signals. Handbook of interpersonal communication 2 (1994), 229-285.

[7] Rehj Cantrell, Paul Schermerhorn, and Matthias Scheutz. 2011. Learning actions from human-robot dialogues. In 2011 RO-MAN. IEEE, 125-130.

[8] Colleen M Carpinella, Alisa B Wyman, Michael A Perez, and Steven J Stroessner. 2017. The Robotic Social Attributes Scale (RoSAS) Development and Validation. In Proceedings of the 2017 ACM/IEEE International Conference on human-robot interaction. 254-262.

[9] Elizabeth Cha, Yunkyung Kim, Terrence Fong, Maja J Mataric, et al. 2018. A survey of nonverbal signaling methods for non-humanoid robots. Foundations and Trends® in Robotics 6, 4 (2018), 211-323.

[10] Susan Wagner Cook, Terina Kuangyi Yip, and Susan Goldin-Meadow. 2012. Gestures, but not meaningless movements, lighten working memory load when explaining math. Language and cognitive processes 27, 4 (2012), 594-610.

[11] Martin J Crowder. 2017. Analysis of repeated measures. Routledge.

[12] Antonella De Angeli, Walter Gerbino, Giulia Cassano, and Daniela Petrelli. 1998. Visual display, pointing, and natural language: the power of multimodal interaction. In Proceedings of the working conference on Advanced visual interfaces. 164-173.

[13] Ewart J De Visser, Samuel S Monfort, Ryan McKendrick, Melissa AB Smith, Patrick E McKnight, Frank Krueger, and Raja Parasuraman. 2016. Almost human: Anthropomorphism increases trust resilience in cognitive agents. Fournal of Experimental Psychology: Applied 22, 3 (2016), 331.

[14] Susan Goldin-Meadow. 1999. The role of gesture in communication and thinking. Trends in cognitive sciences 3, 11 (1999), 419-429.

[15] Thomas Groechel, Zhonghao Shi, Roxanna Pakkar, and Maja J Matarić. 2019. Using Socially Expressive Mixed Reality Arms for Enhancing Low-Expressivity 
Robots. In 2019 28th IEEE International Conference on Robot and Human Interactive Communication (RO-MAN). IEEE, 1-8.

[16] Peter A Hancock, Deborah R Billings, Kristin E Schaefer, Jessie YC Chen, Ewart J De Visser, and Raja Parasuraman. 2011. A meta-analysis of factors affecting trust in human-robot interaction. Human factors 53, 5 (2011), 517-527.

[17] Marcel Heerink, Ben Kröse, Vanessa Evers, and Bob Wielinga. 2010. Assessing acceptance of assistive social agent technology by older adults: the almere model International journal of social robotics 2, 4 (2010), 361-375.

[18] Bertram F Malle and Matthias Scheutz. 2014. Moral competence in social robots. In 2014 IEEE international symposium on ethics in science, technology and engineering. IEEE, $1-6$.

[19] William Marslen-Wilson, Elena Levy, and Lorraine K Tyler. 1982. Producing interpretable discourse: The establishment and maintenance of reference. Speech, place, and action (1982), 339-378.

[20] S. Mathôt. 2017. Bayes like a Baws: Interpreting Bayesian repeated measures in JASP [Blog Post]. https://www.cogsci.nl/blog/interpreting-bayesian-repeatedmeasures-in-jasp.

[21] Nikolaos Mavridis. 2015. A review of verbal and non-verbal human-robot interactive communication. Robotics and Autonomous Systems 63 (2015), 22-35.

[22] RD Morey and JN Rouder. 2014. BayesFactor (Version 0.9. 9).

[23] Raedy Ping and Susan Goldin-Meadow. 2010. Gesturing saves cognitive resources when talking about nonpresent objects. Cognitive Science 34, 4 (2010), 602-619.

[24] Laurel D Riek, Philip C Paul, and Peter Robinson. 2010. When my robot smiles at me: Enabling human-robot rapport via real-time head gesture mimicry. fournal on Multimodal User Interfaces 3, 1-2 (2010), 99-108.

[25] Paul Robinette, Ayanna M Howard, and Alan R Wagner. 2015. Timing is key for robot trust repair. In International Conference on Social Robotics. Springer, 574-583.

[26] Jeffrey N Rouder, Richard D Morey, Paul L Speckman, and Jordan M Province. 2012 Default Bayes factors for ANOVA designs. Fournal of Mathematical Psychology 56, 5 (2012), 356-374.

[27] Maha Salem, Friederike Eyssel, Katharina Rohlfing, Stefan Kopp, and Frank Joublin. 2013. To err is human (-like): Effects of robot gesture on perceived anthropomorphism and likability. International fournal of Social Robotics 5, 3 (2013), 313-323

[28] Maha Salem, Stefan Kopp, Ipke Wachsmuth, Katharina Rohlfing, and Frank Joublin. 2012. Generation and evaluation of communicative robot gesture. International fournal of Social Robotics 4, 2 (2012), 201-217.

[29] Allison Sauppé and Bilge Mutlu. 2014. Robot deictics: How gesture and context shape referential communication. In 2014 9th ACM/IEEE International Conference on Human-Robot Interaction (HRI). IEEE, 342-349.

[30] Matthias Scheutz, Paul Schermerhorn, James Kramer, and David Anderson. 2007. First steps toward natural human-like HRI. Autonomous Robots 22, 4 (2007), 411-423.

[31] Adam Stogsdill, Thao Phung, and Tom Williams. 2020. Investigating ConfidenceBased Category Transition of Spatial Gestures. In Proceedings of the 2nd Workshop on Natural Language Generation for Human-Robot Interaction at HRI 2020.

[32] Daniel Szafir, Bilge Mutlu, and Terrence Fong. 2015. Communicating directionality in flying robots. In 2015 10th ACM/IEEE International Conference on Human-Robot Interaction (HRI). IEEE, 19-26.

[33] JASP Team. 2018. JASP (Version 0.8.5.1)[Computer software].

[34] Stefanie Tellex, Nakul Gopalan, Hadas Kress-Gazit, and Cynthia Matuszek. 2020 Robots That Use Language. Annual Review of Control, Robotics, and Autonomous Systems 3 (2020).

[35] Nhan Tran, Kai Mizuno, Trevor Grant, Thao Phung, Leanne Hirshfield, and Tom Williams. 2019. Exploring Mixed Reality Robot Communication Under Different types of Mental Workload. In Proceedings of the 3rd International Workshop on Virtual, Augmented, and Mixed Reality for HRI.

[36] EJ Wagenmakers, J Love, M Marsman, T Jamil, A Ly, and J Verhagen. 2018 Bayesian inference for psychology, Part II: Example applications with JASP. Psychonomic Bulletin and Review 25, 1 (2018), 35-57.

[37] Tom Williams, Matthew Bussing, Sebastian Cabrol, Elizabeth Boyle, and Nhan Tran. 2019. Mixed Reality Deictic Gesture for Multi-Modal Robot Communication. In Proceedings of the 14th ACM/IEEE International Conference on Human-Robot Interaction.

[38] Tom Williams, Matthew Bussing, Sebastian Cabrol, Ian Lau, Elizabeth Boyle, and Nhan Tran. 2019. Investigating the Potential Effectiveness of Allocentric Mixed Reality Deictic Gesture. In Proceedings of the 11th International Conference on Virtual, Augmented, and Mixed Reality.

[39] Tom Williams and Matthias Scheutz. 2016. A Framework for Resolving OpenWorld Referential Expressions in Distributed Heterogeneous Knowledge Bases. In Proceedings of the 30th AAAI Conference on Artificial Intelligence.

[40] Tom Williams and Matthias Scheutz. 2019. Reference in Robotics: A Givennes Hierarchy Theoretic Approach. In The Oxford Handbook of Reference, Jeanette Gundel and Barbara Abbott (Eds.).

[41] Tom Williams, Daniel Szafir, and Tathagata Chakraborti. 2019. The RealityVirtuality Interaction Cube. In Proceedings of the 2nd International Workshop on Virtual, Augmented, and Mixed Reality for HRI
[42] Tom Williams, Daria Thames, Julia Novakoff, and Matthias Scheutz. 2018. "Thank You for Sharing that Interesting Fact!": Effects of Capability and Context on Indirect Speech Act Use in Task-Based Human-Robot Dialogue. In Proceedings of the 13th ACM/IEEE International Conference on Human-Robot Interaction.

[43] Tom Williams, Nhan Tran, Josh Rands, and Neil T. Dantam. 2018. Augmented, Mixed, and Virtual Reality Enabling of Robot Deixis. In Proceedings of the 10th International Conference on Virtual, Augmented, and Mixed Reality. 